estimated the number of referred patients, $60 \%$ saw more than $>5$ per month. Of centres with no policy only $26 \%$ estimated that they received $>5$ referrals per month. Without a policy $72 \%$ of referrals came from clinical suspicion alone.

Overall $96 \%$ of respondents felt that all patients at high risk of OSA should be screened for OSA. 36 respondents thought it would be ethical to randomise identified cases of OSA to a potential trial of peri-operative CPAP or no CPAP, compared with 40 who did not.

Conclusions There is no established UK standard practice for screening for OSA pre-operatively, despite a majority opinion amongst questionnaire responders that high risk patients should be. There would be cost implications if National pre-operative OSA screening was implemented and there therefore needs to be clear evidence based benefit before proceeding.

\section{S24 REPEATABILITY AND EFFECT OF INCENTIVES ON AN OFFICE BASED ADVANCED DRIVING SIMULATOR (MINIUOLDS) TO ASSESS DRIVING PERFORMANCE IN OBSTRUCTIVE SLEEP APNOEA SYNDROME (OSAS)}

${ }^{1} \mathrm{~A}$ Dwarakanath, ${ }^{2} \mathrm{SL}$ Jamson, ${ }^{3} \mathrm{PD}$ Baxter, 'MW Elliott. 'St. James' University Hospital, Leeds, UK; ${ }^{2}$ Institute for Transport Studies, University of Leeds, Leeds, UK; ${ }^{3}$ Division of Biostatistics, LIGHT, Centre for Epidemiology and Biostatistics, University of Leeds, Leeds, UK

\subsection{6/thoraxjnl-2014-206260.30}

Introduction Some patients with OSAS are at higher risk of being involved in road traffic accidents. No objective tests have been shown to predict reliably whether an individual is safe to drive or not and there is significant variation in the advice given by the clinicians. Using continuously measured variables in an advanced PC-based driving simulator the at risk patients can be identified with a high degree of accuracy.

We have investigated whether this finding is repeatable. Individuals may "raise their game" if they know that their licence is at stake. We have therefore also investigated the effect of an incentive on the test.

Methods 150 untreated OSAS patients (males-131) were randomised to either the repeatability $(\mathrm{n}=50)$ or incentive arm $(\mathrm{n}=$ 100). All performed a simulator run, after initial acclimatisation. In the repeatability arm, patients performed the simulator run on two separate occasions with no knowledge of the results. In the incentive arm, patients performed the simulator run on two separate occasions but just prior to the second run were told about their performance and offered a prize if they could improve their performance by $10 \%$.

SDLP in epoch 3 and "veer" reaction time (Veer-RT) were the co-primary outcome variables. Classification of patients into "pass", "fail" and "indeterminate" were the secondary outcome variables. Results were analysed using paired and unpaired $\mathrm{T}$ tests with the level of significance set at $\mathrm{p}<0.05$.

Results 137 patients (repeatability arm-48, incentive arm-89) completed the trial. The median duration between the two simulator runs was 13 days (range, 5-55). SDLP in epoch 3 and Veer-RT were repeatable (P- 0.54, $\Delta$ SDLP- 0.01 and P- $0.37, \Delta$ Veer-RT- 0.13) respectively. There was no effect of an incentive on SDLP in epoch 3 (P-0.18) and Veer-RT (P-0.57). There was no difference in the simulator outcome between the two runs [pass (P- 0.70), indeterminate (0.06), fail (P- 0.16)].

Conclusions SDLP and Veer-RT are consistent between runs on the MiniUoLDS and this is not affected by a simple incentive. Advanced office PC based simulators may be helpful when advising patients with OSAS about driving.
S25 SLEEPY SNORERS WITH "FLOW LIMITATION SYNDROME": A MISSED OPPORTUNITY FOR CPAP?

${ }^{1} \mathrm{R}$ Yadavilli, 'B Chakrabarti, 'S McDougall, ${ }^{2} \mathrm{~L}$ Horne, 'S Emegbo, ${ }^{1} \mathrm{~S}$ Craig, ${ }^{1} \mathrm{~N}$ Duffy, ${ }^{1} \mathrm{R}$ Parker, ${ }^{1} \mathrm{~J}$ O'Reilly. 'Aintree Chest Centre, University Hospital Aintree, Liverpool, UK; 'University of Liverpool, Liverpool, UK

\subsection{6/thoraxjnl-2014-206260.31}

Background The apnoea-hypopnoea index (AHI) is used to define Obstructive Sleep Apnoea Syndrome (OSAS). Some subjects however, present primarily with excessive daytime sleepiness (EDS) and loud snoring, but investigation may reveal an elevated Respiratory Disturbance Index (RDI) with most events comprising Flow limitations. Little UK based data exists regarding treatment outcomes in this group.

Methodology/results 118 subjects (mean age 52 years; Epworth sleepiness scale score (ESS) 13.58 (5.30); 80\% male) presented between November 2011-October 2013 to the Sleep Service with EDS as a primary symptom, loud snoring, RDI $>15$ with AHI $\leq 11$ (Mean RDI 21.77 (9.43)); AHI 8.03(2.74); ODI 6.72 (4.49) and were treated with CPAP. At 30 day compliance review, 60\% (71/118) had benefited from CPAP with mean ESS pre-CPAP 14.13 (5.12) falling to 7.70 (4.82) following CPAP. The mean BMI was found to be significantly higher in those 71 subjects benefiting from CPAP (33.20 (SD 8.13) v 30.26 (SD 7.40); p = 0.04) but no significant differences were noted in baseline Epworth score, age, gender, AHI, RDI, ODI and Pulse Transit Time (PTT).

This "Flow Limitation" cohort was compared with 261 subjects (mean age 56 years; ESS 12.47(5.61); 82\%Male) diagnosed with OSAS during the same time period (Mean AHI 37.11 (19.94); mean ODI 31.15 (19.74) and treated with CPAP. 76\% (199/261) of the OSAS group reported benefit from CPAP; ESS fell from 13.24 (5.35) to 6.60 (4.74) following CPAP therapy.

Comparing the "Flow Limitation" group with the "OSA" group, the mean BMI (32.03(7.94) v 34.70(8.65); p = 0.004) and age $(51.75(12.34)$ v 56.20(12.18); $\mathrm{p}=0.001)$ were significantly lower in the "Flow Limitation" subjects but no significant difference was noted in baseline ESS. Those deriving benefit from CPAP in the OSA group demonstrated significantly higher CPAP usage $(4.45(2.24)$ v $3.83(2.15)$ hours/night; $\mathrm{p}=0.04)$.

Conclusion Basing treatment decisions on AHI rather than RDI may miss a proportion of patients exhibiting similar levels of EDS as those with OSAS who would otherwise have gained benefit from CPAP. Despite the observed benefit, CPAP usage appeared lower in this "Flow Limitation" cohort who appeared overall to be a younger group with a lower BMI compared to those with OSA.

\section{S26 WHAT ARE THE PREDICTORS OF DEVELOPING HYPOVENTILATION IN OBESITY?}

${ }^{1}$ A Manuel, ${ }^{2} \mathrm{~N}$ Hart, ${ }^{1} \mathrm{~J}$ Stradling. 'Oxford Centre for Respiratory Medicine, Oxford Biomedical Research Centre, Churchill Campus, Oxford University Hospitals NHS Trust, Oxford, OX3 7LJ, UK, Oxford, UK; '² Lane Fox Clinical Respiratory Physiology Centre, St Thomas' Hospital, London, UK

\subsection{6/thoraxjnl-2014-206260.32}

Introduction Obesity Hypoventilation Syndrome (OHS) is conventionally defined by the combination of obesity (BMI $>30 \mathrm{~kg}$ / $\left.\mathrm{m}^{2}\right)$ and daytime hypercapnia $\left(\mathrm{PaCO}_{2}>6 \mathrm{kPa}\right.$, with no alternative explanation); sleep-disordered breathing may or may not be included in the definition. The development of ventilatory failure in obese individuals is highly variable, and the additional factors responsible have not been comprehensively studied. In obese 


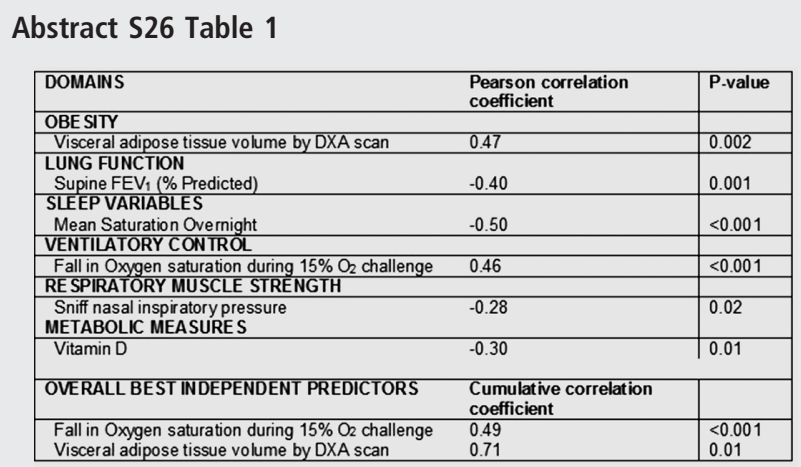

The table shows the correlation coefficient for the statistically strongest predictors of a raised $B E$ in each domain by multiple linear regression. The bottom of the table shows the overall outcome of the multiple linear regression, when each of the strongest independent predictors were matched against each other.

individuals, the presence of a raised plasma standard bicarbonate (or base excess, $\mathrm{BE}$ - a biomarker of whole body acid-base balance, including overnight $\mathrm{PaCO}_{2}$ levels), without necessarily a raised daytime $\mathrm{PaCO}_{2}$, has been shown by us to be an intermediary stage towards overt obesity-hypoventilation syndrome. Thus we have looked for biologically plausible predictors of a raised base excess in obesity, whether or not there was also a raised $\mathrm{PaCO}_{2}$ awake.

Methods 78 obese subjects (BMI $>30$, mean 47 (SD 10, range 32 to 74$) \mathrm{kg} / \mathrm{m}^{2}$ ) were identified from a variety of sources, regardless of their $\mathrm{PaCO} 2$ and acid/base status (mean levels 5.6 (SD 0.8, range 4.2 to 9.6 ) $\mathrm{kPa}$; and 2.1 (SD 2.4, range -3.5 to 10) $\mathrm{mmol} / \mathrm{l}$ respectively) and a large number of their characteristics measured. Biological plausible domains were constructed that were thought potentially to contribute to any ventilatory failure. First, the best independent predictor of the $\mathrm{BE}$ within each domain was found, second, the best overall independent predictors were found. The domains were as follows:

- Obesity and its distribution (BMI, simple surface measures, DXA [a radiographic derivative])

- Lung function (sitting/lying spirometry and forced oscillometry)

- Sleep variables (AHI, ODI, mean overnight $\mathrm{SaO} 2$, time below 90\% SaO2)

- Ventilatory control (2 point responses to $15 \%$ O2 and 5\% $\mathrm{CO} 2)$

- Respiratory muscle strength (mouth pressures, sniff pressures)

- Metabolic measures (e.g. leptin, adipokines, vitamin D)

Conclusions There are a number of strong predictors for the presence of a raised base excess in obesity. Significant predictors were found in each of the biological domains we studied, suggesting that the cause of ventilatory failure in obesity is likely to be multifactorial. However, reduced hypoxic poikilocapnic ventilatory drive and the presence of intra-abdominal obesity seem to be the most powerful predictors of a raised base excess in obesity.

\section{S27 VENOUS BICARBONATE AS A CLINICAL TOOL FOR IDENTIFYING OBESITY HYPOVENTILATION SYNDROME IN THE SLEEP CLINIC}

B Prudon, SD West. Newcastle Regional Sleep Service, Newcastle Upon Tyne, UK

10.1136/thoraxjnl-2014-206260.33

Introduction Obesity Hypoventilation Syndrome (OHS) is defined as sleep disordered breathing, obesity, and daytime hypercapnia, without another cause of ventilatory impairment.
Literature suggests $10-25 \%$ of patients assessed for Obstructive Sleep Apnoea (OSA) have OHS, with significantly increased morbidity and mortality. Early identification may be beneficial. Studies suggest venous bicarbonate $\left(\mathrm{vHCO}_{3}{ }^{-}\right) \geq 27 \mathrm{mmol} / \mathrm{l}$ can be used to screen for OHS. We assessed the impact of incorporating this measurement into patient assessments.

Methods Obese out-patients referred for possible OSA had $\mathrm{vHCO}_{3}{ }^{-}$measured. Patients with a $\mathrm{vHCO}_{3}{ }^{-} \geq 27 \mathrm{mmol} / \mathrm{l}$ underwent arterial blood gas (ABG) analysis. Those with $\mathrm{pCO}_{2}>6.2$ $\mathrm{kPa}$ underwent further assessments to identify the cause of ventilatory impairment. None had been referred specifically for investigation of OHS. Patients had domiciliary or in-patient sleep studies as per standard practice.

Results There were 288 patients included: 65\% males, mean (SD) age 50 years (range 21-79 years), BMI $39.2 \mathrm{~kg} / \mathrm{m}^{2}$ (7.8), Epworth Sleepiness Scale 13 (6), daytime $\mathrm{SpO}_{2}$ on air 97\% (2.1). Sleep study results showed the Apnoea-Hypopnea Index (AHI) to be $\geq 5$ in $88 \%$, and $\geq 30$ in $49 \%$. Mean $\mathrm{vHCO}_{3}{ }^{-}$was $26.2 \mathrm{mmol} / \mathrm{l}(2.7) . \mathrm{vHCO}_{3}{ }^{-}$correlated significantly $(\mathrm{r}=0.3-0.4$, $\mathrm{p}<0.005)$ with daytime $\mathrm{SpO}_{2}$, mean overnight $\mathrm{SpO}_{2}$, time spent $<80 \%$ and $<90 \%$, but not AHI or ODI.

$\mathrm{vHCO}_{3}{ }^{-}$was $\geq 27 \mathrm{mmol} / \mathrm{l}$ in 123 (43\%), of whom 80 had an ABG measurement; mean $\mathrm{pCO}_{2} 5.4 \mathrm{kPa}(0.8)$, ten patients $>6.2$ $\mathrm{kPa}$. Ventilatory impairment was due to $\mathrm{OHS}$ in four $(5 \%$ of ABG cohort); there was additional lung or chest wall disease in the other six. Overall, 25 patients had a base excess $\geq 3$. The $\mathrm{vHCO}_{3}{ }^{-}$range was $28-36 \mathrm{mmol} / \mathrm{l}$ in patients with $\mathrm{OHS}$, with a BMI range of $38-53 \mathrm{~kg} / \mathrm{m}^{2}$.

Three additional outpatients with BMI $>50 \mathrm{~kg} / \mathrm{m}^{2}$ were diagnosed with $\mathrm{OHS}$ on $\mathrm{ABG}$ without $\mathrm{vHCO}_{3}{ }^{-}$measurement. In all seven OHS patients, CPAP was initiated. One was non-compliant, four improved and two required home non-invasive ventilation due to non-improvement in ABG.

Conclusions In this large cohort of patients assessed for OSA, $43 \%$ had a $\mathrm{vHCO}_{3}^{-} \geq 27 \mathrm{mmol} / \mathrm{l}$ indicating possible OHS, but only $5 \%$ were actually diagnosed with OHS. In isolation this strategy to identify OHS seems inefficient. An increased $\mathrm{vHCO}_{3}{ }^{-}$ in combination with sleep study data may be superior.

\section{S28 LIRAGLUTIDE 3.0 MG REDUCES SEVERITY OF OBSTRUCTIVE SLEEP APNOEA AND BODY WEIGHT IN OBESE INDIVIDUALS WITH MODERATE OR SEVERE DISEASE: SCALE SLEEP APNOEA TRIAL}

${ }^{1} \mathrm{~A}$ Collier, ${ }^{2} \mathrm{~A}$ Blackman, ${ }^{3} \mathrm{G}$ Foster, ${ }^{4} \mathrm{G}$ Zammit, ${ }^{5} \mathrm{R}$ Rosenberg, ${ }^{6} \mathrm{~T}$ Wadden, ${ }^{7} \mathrm{~L}$ Aronne, ${ }^{8} \mathrm{~B}$ Claudius, ${ }^{8} \mathrm{~T}$ Jensen, ${ }^{9} \mathrm{E}$ Mignot. ${ }^{7}$ Ayr Hospital, Ayr, UK; ${ }^{2}$ Toronto Sleep Institute, MedSleep, Toronto, Canada; ${ }^{3}$ Temple University, Philadelphia, USA; ${ }^{4}$ Clinilabs Sleep Disorders Institute, New York, USA; ${ }^{5}$ NeuroTrials Research, Atlanta, USA; ${ }^{6}$ University of Pennsylvania, Philadelphia, USA; ${ }^{7}$ Weill Cornell Medical College, New York, USA; ${ }^{8}$ Novo Nordisk A/S, Soborg, Denmark; ${ }^{9}$ Stanford University, Palo Alto, USA

\subsection{6/thoraxjnl-2014-206260.34}

Aims/objectives This randomised, double-blind, parallel-group trial compared the effects of liraglutide $3.0 \mathrm{mg}$ to placebo, both as adjunct to diet and exercise, on obstructive sleep apnoea (OSA) severity and body weight.

Content Obese individuals $(\mathrm{n}=359)$ without diabetes who had moderate or severe OSA and were unwilling/unable to use continuous positive airway pressure therapy were randomised 1:1 to liraglutide $3.0 \mathrm{mg}$ or placebo for 32 weeks (baseline characteristics: 48.5 years, males $71.9 \%$, apnoea-hypopnoea index [AHI] 49.2 events/h, body weight $117.6 \mathrm{~kg}$, BMI $\left.39.1 \mathrm{~kg} / \mathrm{m}^{2}, \mathrm{HbA}_{1 \mathrm{c}} 5.7 \%\right)$. Outcomes At end-of-trial, the reduction in AHI was significantly greater with liraglutide $3.0 \mathrm{mg}$ than placebo (Table). Liraglutide 